ARTICLE

\title{
Towards single-chip radiofrequency signal processing via acoustoelectric electron-phonon interactions
}

Lisa Hackett ${ }^{1}$, Michael Miller ${ }^{1}$, Felicia Brimigion ${ }^{1}$, Daniel Dominguez ${ }^{1}$, Greg Peake${ }^{1}$, Anna Tauke-Pedretti ${ }^{1}$, Shawn Arterburn ${ }^{1}$, Thomas A. Friedmann ${ }^{1} \&$ Matt Eichenfield (iD ${ }^{1 \times}$

The addition of active, nonlinear, and nonreciprocal functionalities to passive piezoelectric acoustic wave technologies could enable all-acoustic and therefore ultra-compact radiofrequency signal processors. Toward this goal, we present a heterogeneously integrated acoustoelectric material platform consisting of a $50 \mathrm{~nm}$ indium gallium arsenide epitaxial semiconductor film in direct contact with a $41^{\circ} \mathrm{YX}$ lithium niobate piezoelectric substrate. We then demonstrate three of the main components of an all-acoustic radiofrequency signal processor: passive delay line filters, amplifiers, and circulators. Heterogeneous integration allows for simultaneous, independent optimization of the piezoelectric-acoustic and electronic properties, leading to the highest performing surface acoustic wave amplifiers ever developed in terms of gain per unit length and DC power dissipation, as well as the first-ever demonstrated acoustoelectric circulator with an isolation of $46 \mathrm{~dB}$ with a pulsed DC bias. Finally, we describe how the remaining components of an all-acoustic radiofrequency signal processor are an extension of this work.

\footnotetext{
${ }^{1}$ Microsystems Engineering, Science, and Applications, Sandia National Laboratories, Albuquerque, NM, USA. ${ }_{\text {email: meichen@sandia.gov }}$
} 
$\mathrm{M}$ odern radiofrequency $(\mathrm{RF})$ signal processors (RFSPs) make use of multiple technologies, integrated at the system level, to achieve all their necessary functionalities. For example, high-performance passive filtering and delay lines are typically achieved in piezoelectric acoustic wave technologies such as lithium niobate $\left(\mathrm{LiNbO}_{3}\right)$ surface acoustic wave $(\mathrm{SAW})^{1-3}$ and aluminum nitride bulk acoustic wave (BAW) $)^{4,5}$ platforms. Active devices such as amplifiers and mixers are typically realized in semiconductor technologies such as a complementary metal oxide semiconductor (CMOS) $)^{6,7}$ and gallium arsenide $(\mathrm{GaAs})^{8,9}$. Nonreciprocity for circulators and isolators is achieved through the gyromagnetic effect via magnetized ferrite material in proximity to $\mathrm{RF}$ transmission lines ${ }^{10,11}$. More recent approaches to nonreciprocal RF devices use spatiotemporal modulation in coupled inductor-capacitor (LC) or acoustic resonators in a ring or wye topology, but require an RF drive for operation $^{12-15}$

While these technologies are all highly optimized for the performance of their respective components, their integration at the system level ultimately hinders the miniaturization of RFSPs, which is in direct conflict with the overarching technological trend toward simultaneous proliferation and miniaturization of $\mathrm{RF}$ systems ${ }^{16}$. The complexity of the RFSP front-end-especially in terms of the number of required filters and amplifiers-will increase significantly with the requirement to support more frequency bands ${ }^{17,18}$ and multiple-input/multiple-output designs ${ }^{19}$ in an increasingly crowded and congested spectral landscape. In addition, simultaneous transmit and receive (STAR) systems where the transmitter $(\mathrm{Tx})$ and receiver $(\mathrm{Rx})$ operate simultaneously in the same frequency band are highly sought after to improve spectral efficiency by essentially doubling the available bandwidth ${ }^{20}$. An all-acoustic RFSP would be ultra-compact, but fundamental components remain to be demonstrated. SAW signal processing can also address a wide range of frequencies from $\mathrm{MHz}$ to $\mathrm{GHz}$ by simply changing the electrode pitch ${ }^{21-23}$. This could allow the RFSP of many frequency bands to be fabricated on a single chip, greatly reducing the size, the number of components, packaging requirements, and assembly required to achieve the complex RFSP circuitry present in modern RF devices.

In this article, we demonstrate three of the necessary fundamental components, fabricated on the same chip, to realize an allacoustic and therefore ultra-compact RFSP: passive filtering with time delay, amplification, and non-reciprocal circulators, and, in the discussion, we lay out a vision for an all-acoustic RFSP based on the acoustoelectric effect. We present the best performing acoustic amplifier ever in terms of gain per unit length and DC power dissipation, the first-ever acoustoelectric circulator, and the co-fabrication of these active acoustic wave components on the same chip as high performing passive acoustic wave delay lines. We achieve this via heterogeneous integration of piezoelectricacoustic wave and semiconductor materials to produce strong acoustoelectric electron-phonon interactions between the electronic charge carriers in the semiconductor and the electric fields associated with the SAW phonons. Using this platform, we simultaneously realize the exquisite performance of passive piezoelectric acoustic wave devices and selectively endow acoustic circuit elements with nonlinear and nonreciprocal functionality through electron-phonon acoustoelectric interactions ${ }^{24-29}$. Our heterogeneously integrated acoustoelectric platform consists of a $50 \mathrm{~nm}$ epitaxial indium gallium arsenide $\left(\mathrm{In}_{0.53} \mathrm{Ga}_{0.47} \mathrm{As}\right)$ semiconductor layer on a $41^{\circ} \mathrm{YX} \mathrm{LiNbO}_{3}$ substrate that supports a shear-horizontal SAW (SH-SAW) with an electromechanical coupling coefficient $\left(K^{2}\right)$ of $17 \%$ and minimal propagation $\operatorname{losses}^{30}$. We achieve an acoustically thin, high mobility $\mathrm{In}_{0.53} \mathrm{Ga}_{0.47} \mathrm{As}$ semiconductor layer (measured Hall mobility of $2000 \mathrm{~cm}^{2} / \mathrm{V}$-s); with a thickness of only $50 \mathrm{~nm}$, the devices can provide acoustoelectric interactions out to at least $5 \mathrm{GHz}^{31}$. Furthermore, the low silicon $(\mathrm{Si})$ doping density of electrons, $N$, in the semiconductor $\left(1 \times 10^{16} \mathrm{~cm}^{-3}\right)$, made possible due to metal-organic chemical vapor deposition (MOCVD), provides extremely high gain with low ohmic heat dissipation. In addition to passive delay lines on $41^{\circ} \mathrm{YX} \mathrm{LiNbO}_{3}$, we demonstrate a 276 $\mathrm{MHz}$ acoustic wave amplifier with a terminal gain of $13.5 \mathrm{~dB}$ in

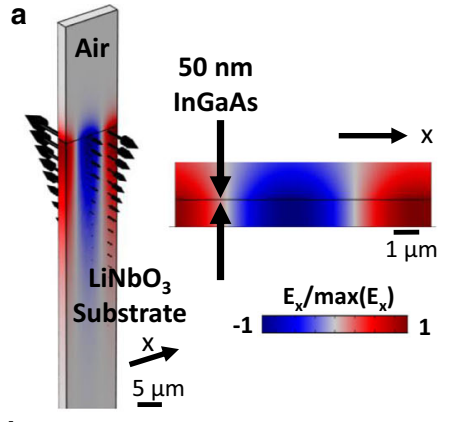

d

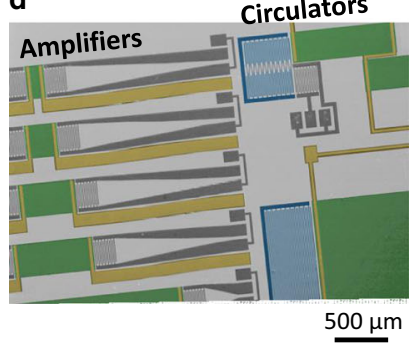

e
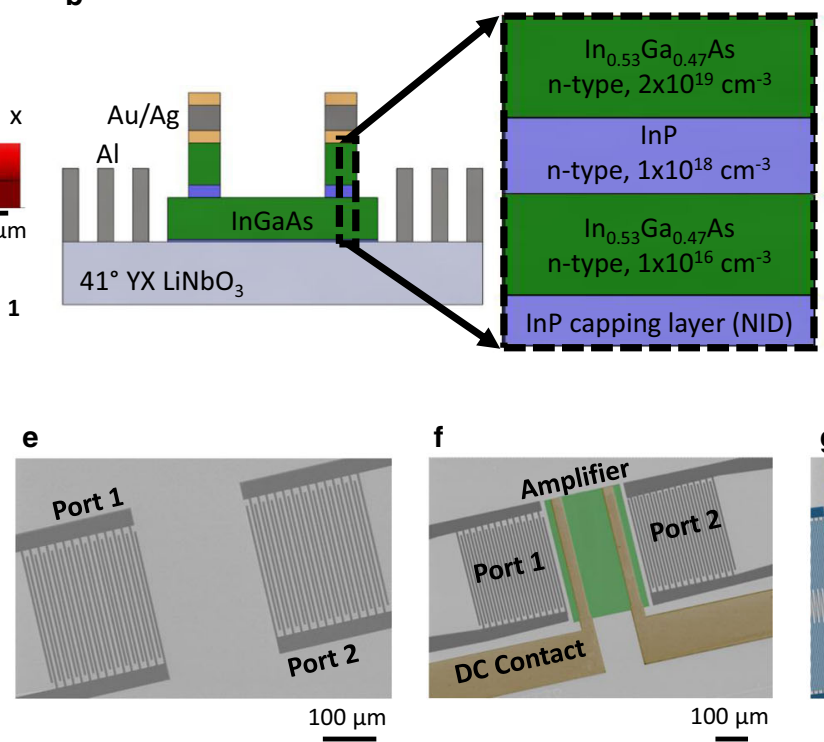

f

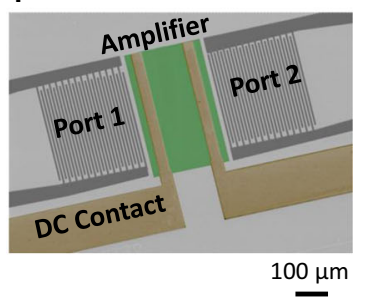

c

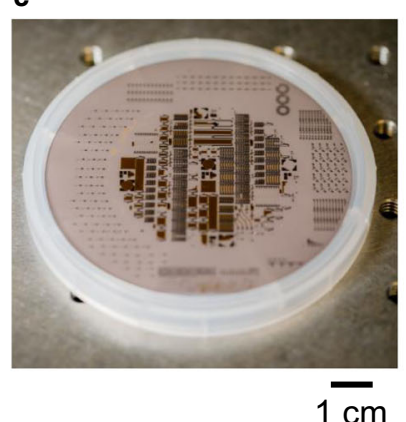

g

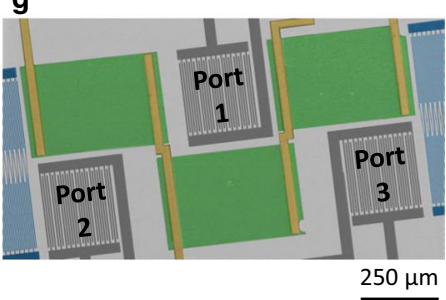

Fig. 1 Material platform overview. a Longitudinal electric field $\left(E_{x}\right)$ from a finite element model, with displacement field indicated by the black arrows, and (b) a schematic of our implementation of the acoustoelectric effect for an $\operatorname{In}_{0.53} \mathrm{Ga}_{0.47} \mathrm{As}$ thin film on a $\mathrm{LiNbO}_{3}$ substrate. c Image of the heterostructure wafer after processing. d False-colored SEM images of a wafer with passive and active acoustic wave fabricated devices including (e) delay lines, (f) amplifiers, and ( $\mathbf{g}$ ) circulators. In the false-colored images, the $\mathrm{LiNbO}_{3}$ substrate is light gray, the $\ln _{0.53} \mathrm{Ga}_{0.47}$ As thin film is green, the RF transducers are dark gray, the DC contacts are gold, and the RF couplers are blue. 
$505 \mu \mathrm{m}$ that dissipates only $15 \mathrm{~mW}$ of DC power and the firstever nonmagnetic acoustoelectric circulator that requires only a DC bias for operation with an isolation of $46 \mathrm{~dB}$.

\section{Results}

Material platform overview. By heterogeneously integrating and selectively removing epitaxial semiconductor layers on a piezoelectric substrate, we selectively create active and nonreciprocal acoustic devices through the acoustoelectric effect on the same wafer on which we fabricate high performance passive piezoelectric acoustic devices. The acoustoelectric effect arises in our platform due to charge bunching mediated by the interaction between charge carriers in the semiconductor and the evanescent electric field that accompanies the piezoelectric SAW ${ }^{26}$. Depending on the charge carrier drift velocity $v_{d}$, controlled by an applied drift field $E_{d}$, the bunched charge either lags or leads the acoustic wave. The gain mechanism, which is analogous to an electromagnetic traveling-wave tube amplifer ${ }^{32}$, depends on the average interaction of the charge carriers with the piezoelectric electric field. This interaction controls the energy transfer from or to the acoustic wave, resulting in acoustic wave attenuation or amplification, respectively. The simulated longitudinal electric field and mechanical displacement for the system we study here, which is a $50 \mathrm{~nm} \mathrm{In}_{0.53} \mathrm{Ga}_{0.47} \mathrm{As}$ thin film on a $41^{\circ} \mathrm{YX} \mathrm{LiNbO}_{3}$ substrate supporting an SH-SAW, is shown in Fig. 1a. The evanescent electric field of the piezoelectric acoustic wave has a strong overlap with the $\operatorname{In}_{0.53} \mathrm{Ga}_{0.47} \mathrm{As}$ thin film in this material system resulting in strong coupling between the electrons and phonons.

While detailed models of acoustic gain and nonreciprocity can give a complete picture of the electron-phonon interaction ${ }^{33,34}$, under the appropriate approximations these models are wellcaptured by a simple, intuitive model that can be derived from an equivalent RC circuit $^{35}$. The acoustic loss $\alpha$ is given by

$$
\alpha=\frac{1}{2} k_{A E}^{2} k_{0} \frac{\gamma \omega \tau}{1+(\gamma \omega \tau)^{2}}
$$

where $k_{A E}^{2}$ is a coupling coefficient describing the interaction strength between the free electrons and the electric field of the piezoelectric acoustic wave, $k_{0}$ is the acoustic wavenumber, $\gamma=1-\left(v_{d} / v_{a}\right), v_{a}$ is the acoustic velocity, $v_{d}=\mu E_{d}$ where $\mu$ is the mobility, and $\tau$ is given by $\tau=\frac{\varepsilon_{0}+\varepsilon_{p}}{k_{0} \sigma t}$ where $\sigma t$ is the semiconductor conductivity-thickness product, $\varepsilon_{0}$ is the permittivity of free space, and $\varepsilon_{p}$ is the piezoelectric permittivity. The gain of a material system is then parameterized by only $k_{A E}^{2}, \mu$, and $\sigma t$ where $k_{A E}^{2}$ is equivalent to $K^{2}$. Important properties to note are that negative $\alpha$, or acoustic gain, only occurs when $v_{d}$ exceeds $v_{a}$ meaning that a voltage $V_{O \mathrm{~dB}}$ must be applied to reach $0 \mathrm{~dB}$. The acoustoelectric interaction is then inherently nonreciprocal as the acoustic wave must travel in the same direction as the unidirectional charge carrier drift to be amplified. In addition, the maximum achievable loss or gain for a given operating frequency is $\alpha_{\max }=k_{A E}^{2} / 4$ and therefore only depends on $k_{A E}^{2}$.

A schematic of our implementation of the acoustoelectric effect as an acoustic amplifier is shown in Fig. 1b. The material platform consists of a heterostructure of an epitaxial $50 \mathrm{~nm} \mathrm{In}_{0.53} \mathrm{Ga}_{0.47} \mathrm{As}$ semiconductor, grown by MOCVD, bonded and processed on the wafer-scale to a $41^{\circ}$ YX $\mathrm{LiNbO}_{3}$ substrate. The $\operatorname{In}_{0.53} \mathrm{Ga}_{0.47}$ As can be made acoustically thin when combined with our process where thicker layers with various doping concentrations, $N$, including layers that are not intentionally doped (NID), are selectively removed. Figure 1c shows an image of the $\mathrm{In}_{0.53} \mathrm{Ga}_{0.47} \mathrm{As} / \mathrm{LiNbO}_{3}$ heterostructure wafer following fabrication while Fig. $1 \mathrm{~d}$ shows a false-colored SEM image of acoustic amplifiers and circulators.
Excluding the $\operatorname{In}_{0.53} \mathrm{Ga}_{0.47} \mathrm{As}$, by selectively etching it away, enables delay line filters and resonators in the underlying piezoelectric technology. Additional false-colored SEM images of an acoustic delay line, amplifier, and circulator are shown in Fig. 1e-g, respectively (see Supplementary Note 1 and Supplementary Figs. 1-2 for additional details on the fabrication process flow and optical microscope device images).

While high-performance delay line filters are already ubiquitous in RF signal processing, here we demonstrate, on the same chip, high-performance acoustoelectric amplifiers and the firstever acoustoelectric circulator. The performance demonstrated herein-in terms of terminal gain, nonreciprocity, and dissipated DC power-is achieved due to the combination of exceptional piezoelectric and semiconductor material parameters, achievable here for the first time in a heterogeneously integrated material platform.

The rest of the article proceeds as follows. We begin by discussing the optimization of the electromechanical and acoustoelectric interactions. We then present the three devices experimentally demonstrated here: an acoustic delay line, acoustoelectric amplifier, and acoustoelectric circulator. We then conclude with a discussion of the remaining components necessary to achieve an all-acoustic RFSP and other potential applications of the technology, such as integrated photonics.

Optimization of the electromechanical and acoustoelectric interaction. To operate as a platform for passive and active acoustic wave devices, the material properties of the $\mathrm{In}_{0.53} \mathrm{Ga}_{0.47} \mathrm{As}$ on $\mathrm{LiNbO}_{3}$ heterostructure must be optimized for acoustic wave generation, amplification, and nonreciprocity. All components are improved by a piezoelectric substrate with a large $K^{2}$ for surface-guided waves and low propagation loss. This leads to lower insertion losses and larger operating bandwidths in addition to a larger $k_{A E}^{2}$. For the acoustic modes of interest on commercially available $\mathrm{LiNbO}_{3}$ substrates, the SH-SAW mode on $41^{\circ} \mathrm{YX} \mathrm{LiNbO}_{3}$ has a $K^{2}$ of $17 \%$, is lossless for the open boundary condition, and has a propagation loss of only $0.04 \mathrm{~dB} / \Lambda$ for the shorted boundary condition, where $\Lambda$ is the acoustic wavelength (see Supplementary Note 2 for a summary of the properties of different $\mathrm{LiNbO}_{3}$ substrates). Due to the exceptional $K^{2}$ and relatively low propagation losses, we focus our work on this $\mathrm{SH}$ SAW mode (see Supplementary Note 3 and Supplementary Fig. 3 for a comparison of $k_{A E}^{2}$ between the Rayleigh mode on YZ $\mathrm{LiNbO}_{3}$ and the SH-SAW on $41^{\circ} \mathrm{YX} \mathrm{LiNbO}_{3}$ ).

The importance of the piezoelectric and semiconductor material parameters for acoustoelectric device performance is quantified in Fig. 2a-c. Overall, to improve the gain and DC power dissipation, it is most important to maximize $K^{2}$ and minimize $\sigma t$. Best performance is obtained by maximizing the gain slope, which, from Eq. (1), requires optimization of $K^{2}, \sigma t$, and $\mu$. However, minimizing the DC power dissipation requires a different optimization with respect to $\sigma t$ and $\mu$. Figure $2 \mathrm{a}$ and $\mathrm{b}$ show contour plots of the gain slope in $\mathrm{dB} / \mathrm{V}$ as a function of $\{\sigma t$ and $\left.K^{2}\right\}$ and $\{\sigma t$ and $\mu$, respectively. The highest gain slope is achieved when $K^{2}$ is maximized and $\sigma t$ is minimized while increasing $\mu$ has no impact only if there is no corresponding increase in $\sigma t$. To maintain the same gain slope with a larger $\mu, \sigma t$ must be reduced by a lower $N$ or thinner semiconductor film. Figure $2 \mathrm{c}$ shows a contour plot of the dissipated DC power $\left(P_{d}\right)$ to achieve $25 \mathrm{~dB}$ of gain as a function of $\sigma t$ and $\mu$ for an acoustoelectric amplifier with a length $(l)$ of $500 \mu \mathrm{m}$ and width (w) of $328 \mu \mathrm{m}$ where $P_{d}=\frac{V^{2} w \sigma t}{l}$. For low $\sigma t, P_{d}$ can be further improved by increasing $\mu$, which decreases $V_{O d B}$. As can be seen from Fig. $2 \mathrm{c}$, there is a range of $\mu$ values that minimize $P_{d}$ for a given $\sigma t$. In our material platform, we achieve a $K^{2}$ of $17 \%$, a $\sigma t$ of 

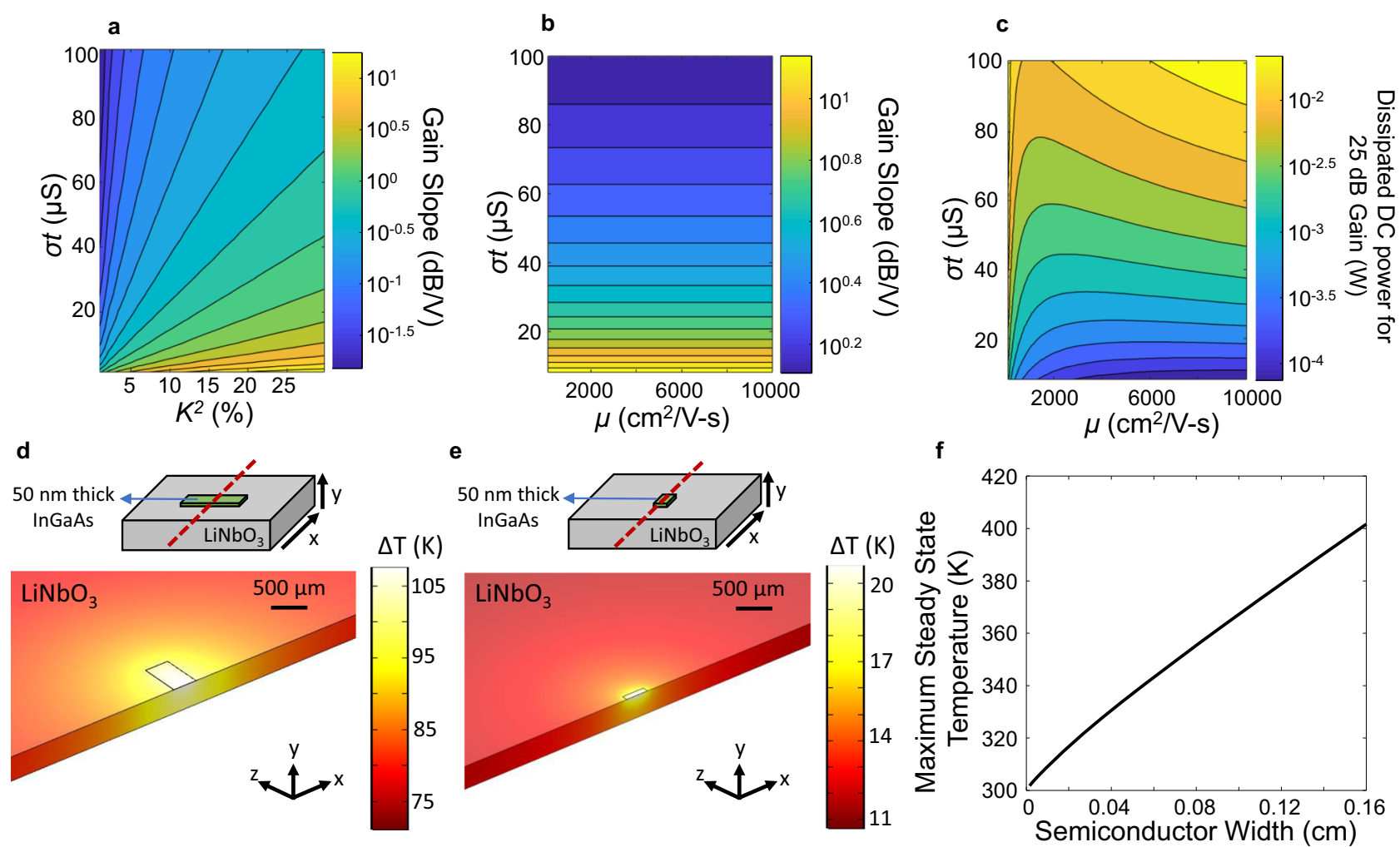

Fig. 2 Material parameters and geometry optimization. Contour plots of the (a) gain slope as a function of $\sigma \mathrm{t}$ and $\mathrm{K}^{2}$ (b) gain slope as a function of $\sigma \mathrm{t}$ and $\mu$, and (c) dissipated DC power for $25 \mathrm{~dB}$ of gain as a function of $\sigma \mathrm{t}$ and $\mu$. The simulated steady-state distribution of the temperature difference ( $\Delta \mathrm{T}$ ) is shown for a semiconductor width of (d) $0.16 \mathrm{~cm}$ and $(\mathbf{e}) 0.024 \mathrm{~cm}$ where the dashed-red line denotes the axis for both the propagation of the acoustic wave and the simulated cross-section along with (f) a plot of the maximum steady-state temperature as a function of semiconductor width from 0.0016 $\mathrm{cm}$ to $0.16 \mathrm{~cm}$

$16 \mu \mathrm{S}$, and mobility of $2000 \mathrm{~cm}^{2} / \mathrm{V}$-s. The specially selected $\mathrm{SH}$ SAW mode on $41^{\circ} \mathrm{YX} \mathrm{LiNbO}_{3}$ with high $K^{2}$ strongly couples the acoustic wave to semiconductor charge carriers while simultaneously having low radiation propagation loss from coupling to bulk modes. Heterogeneous integration of the materials allows for an extremely large interaction strength to be combined with highly optimized semiconductor performance.

Another important consideration for active acoustic wave devices is thermal management, which has historically limited the duty cycle with which amplifiers can be operated ${ }^{24,36}$. The semiconductor layer gives rise to Joule heating and the heat dissipation efficiency depends on convection into the air and thermal conduction into the underlying substrate. The simulated steady-state temperature distribution is shown for a $50 \mathrm{~nm}$ thick $\mathrm{In}_{0.53} \mathrm{Ga}_{0.47} \mathrm{As}$ semiconductor layer with a length of $0.05 \mathrm{~cm}$ and widths of $0.16 \mathrm{~cm}$ (Fig. 2d) and $0.024 \mathrm{~cm}$ (Fig. 2e) on a $\mathrm{LiNbO}_{3}$ chip with a length, width, and thickness of $12.5 \mathrm{~mm} \times 24 \mathrm{~mm} \times$ $0.5 \mathrm{~mm}$, respectively, substrate thermal conductivity of $4.2 \mathrm{~W} /$ $(\mathrm{mK})$, and a heat transfer coefficient of $5 \mathrm{~W} /\left(\mathrm{m}^{2} \mathrm{~K}\right)$. The simulated maximum steady-state temperature as a function of semiconductor width is shown in Fig. 2f. A less wide semiconductor layer is both more resistive-and therefore a smaller overall heat source-and provides more efficient lateral heat dissipation as the heat is efficiently conducted away in the transverse direction. There are situations where the semiconductor width cannot be significantly reduced, for example, because a large aperture is required to achieve a large, diffraction-free propagation length in a delay line. In that case, heat dissipation could also be significantly improved by increasing the thermal conductivity of the substrate, such as using $\mathrm{LiNbO}_{3}$ thin film on $\mathrm{Si}$ instead of a bulk $\mathrm{LiNbO}_{3}$ piezoelectric.
Acoustic delay line. High-performance delay lines were fabricated on $\mathrm{LiNbO}_{3}$ using single-phase unidirectional transducers (SPUDT). Acoustic delay lines with low insertion loss and large operating bandwidth are of high interest for RF applications due to their compact size and ability to incorporate novel passive signal processing functions through the design of the transducer transfer function. Figure 3a shows a schematic of a SAW delay line that uses two traditional interdigitated transducers (IDTs), which have a pitch of $\Lambda / 2$ and an electrode width of $\Lambda / 4$. Figure $3 \mathrm{~b}$ shows a schematic of a SAW delay line that uses two SPUDTs. The traditional IDT results in bidirectional acoustic wave generation, as shown in the simulated displacement field in Fig. $3 c$, which leads to a minimum insertion loss of $6 \mathrm{~dB}$ in a delay line. The SPUDT design uses integrated reflectors with spacings from $\Lambda / 8$ transduction electrodes such that there is constructive interference for the forward propagating wave and destructive interference for the backward propagating wave. As a result, the generation and detection of acoustic waves are unidirectional, ideally reducing the insertion loss by $3 \mathrm{~dB}$ per transducer, as shown in the simulated displacement field in Fig. $3 \mathrm{~d}$.

The measured $S_{21}$ as a function of frequency for a delay line on

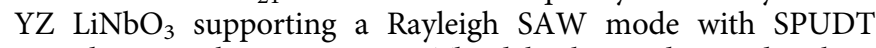
transducers is shown in Fig. 3e. The delay line is designed with $\Lambda$ $=16 \mu \mathrm{m}$, an aperture of $25 \Lambda$, propagation length of $500 \mu \mathrm{m}$, and 45 SPUDT cells. The experimental resonant frequency is 214 $\mathrm{MHz}$, the insertion loss is $4.4 \mathrm{~dB}$, and the fractional bandwidth (FBW) is $1.5 \%$. A plot of the measured $S_{21}$ as a function of frequency for a delay line on $41^{\circ} \mathrm{YX} \mathrm{LiNbO}_{3}$ supporting an $\mathrm{SH}-$ SAW mode with SPUDT transducers is shown in Fig. 3f. The delay line has $\Lambda=16 \mu \mathrm{m}$, an aperture of $15 \Lambda$, propagation length of $250 \mu \mathrm{m}$, and 21 SPUDT cells. The resonant frequency is 276 
a

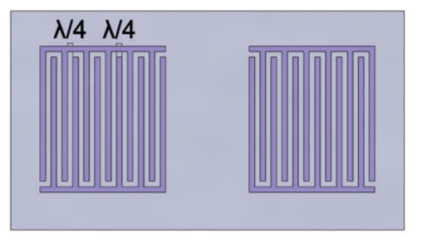

b

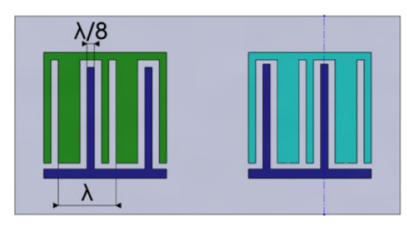

C

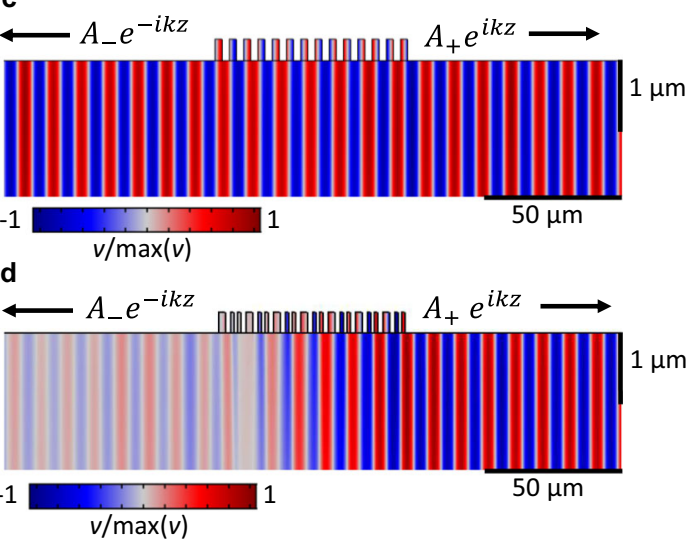

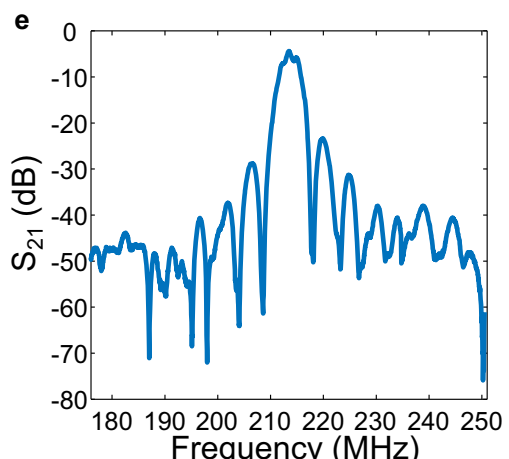

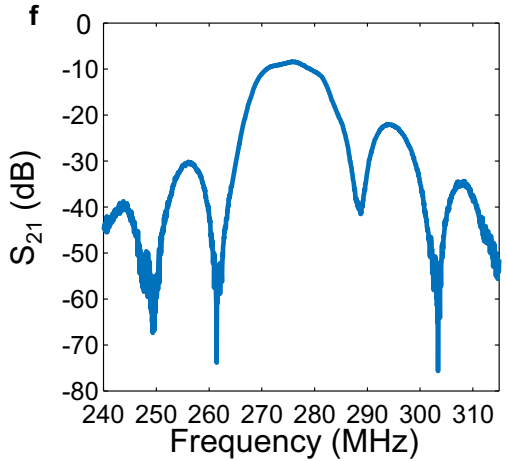

Fig. 3 Acoustic delay line. Schematics of a (a) traditional IDT delay line and a (b) SPUDT design with corresponding simulated normalized displacement fields shown in $\mathbf{c}$ and $\mathbf{d}$, respectively. The $\mathrm{LiNbO}_{3}$ substrate is shown in light gray, the aluminum for the traditional IDTs is shown in light purple, and the aluminum for the SPUDT IDTs is shown in dark purple, green, and cyan. $\lambda$ denotes the acoustic wavelength, $A_{-} e^{-i k z}$ describes the backward propagating wave, $A_{+} e^{i k z}$ describes the forward propagating wave, and $v$ is the displacement along the $y$-axis. Measured $S_{21}$ as a function of frequency for SPUDT delay lines on (e) $\mathrm{YZ} \mathrm{LiNbO}_{3}$ supporting a Rayleigh SAW mode with a resonant frequency of $214 \mathrm{MHz}$ and (f) $41^{\circ} \mathrm{YX} \mathrm{LiNbO}$ supporting an $\mathrm{SH}-\mathrm{SAW}$ mode with a resonant frequency of $276 \mathrm{MHz}$.

$\mathrm{MHz}$, the insertion loss is $8.4 \mathrm{~dB}$, and the FBW is $4.1 \%$. For the delay lines on $\mathrm{YZ}$ and $41^{\circ} \mathrm{YX} \mathrm{LiNbO}_{3}$, matching to the $50 \Omega$ source impedance of the network analyzer was optimized through a two-step process. The approximate number of electrode pairs was calculated using the Mason equivalent circuit model given the IDT aperture, expected capacitance, $K^{2}$, and operating frequency ${ }^{37}$. A bank of delay lines was then fabricated with a varying number of electrode pairs followed by measuring the Sparameters as a function of the number of electrode pairs to determine the optimal number. Previous results have indicated that a more sophisticated transducer design is required to achieve low insertion loss in SH-SAW delay lines compared to Rayleigh waves due to effects such as bulk wave interference ${ }^{38}$. Overall performance for a SPUDT transducer delay line is limited by the fundamental tradeoff between insertion loss and FBW. Ideally, $K^{2}$ and the reflectivity per unit cell will be maximized such that impedance matching and unidirectionality can be achieved with a smaller number of electrodes, which will maximize the FBW while maintaining a low insertion loss. An optimized transducer design will ultimately result in our devices having lower insertion loss that leads to higher performance.

Acoustic amplifier. Voltage-controlled amplification and attenuation of the SH-SAW mode on $41^{\circ} \mathrm{YX} \mathrm{LiNbO}_{3}$ are accomplished by adding a patterned $\mathrm{In}_{0.53} \mathrm{Ga}_{0.47}$ As epitaxial semiconductor with DC contacts to the RF acoustic delay line. The experimental setup for gain measurements is shown in Fig. 4a. The gain is measured by detecting the change in $S_{21}$ with respect to time during a continuous $\mathrm{RF}$ input at $276 \mathrm{MHz}$ and the application of a $1 \mathrm{~ms}$ voltage pulse to the amplifier. The $S_{21}$ value represents the power transmission from the input to the output. Pulsed mode operation is used to avoid thermal drift and runaway, which can corrupt gain measurements. For the acoustoelectric amplifier, the gain bandwidth is limited by the transducer bandwidth.

Here we present our experimental gain data in two ways. One is the electronic gain, which can only be achieved if the amplification is large enough to overcome losses due to the acoustoelectric effect. The other type of gain we present is terminal gain, which can only be achieved once the amplification is large enough to overcome all of the losses in the system, including transducer conversion losses and reflection losses from the thick DC contact metal. Both electronic gain and terminal gain are gains in power (as opposed to amplitude) measured on a network analyzer with a source impedance of $50 \Omega$. A plot of electronic gain in $\mathrm{dB}$ per unit length as a function of drift field is shown in Fig. $4 \mathrm{~b}$ for two devices with a $50 \mathrm{~nm} \mathrm{In}_{0.53} \mathrm{Ga}_{0.47} \mathrm{As}$ amplifier layer and acoustoelectric interaction lengths of $255 \mu \mathrm{m}$ and $505 \mu \mathrm{m}$.

The theoretical gain curve, calculated from Eq. (1), is also shown in Fig. 4(b). The theoretical gain curve is calculated based on a $K^{2}$ of $17 \%, v_{a}$ of $4416 \mathrm{~m} / \mathrm{s}$, operating frequency of $276 \mathrm{MHz}, \mu$ of 2000 $\mathrm{cm}^{2} / \mathrm{V}$-s, and an $N$ of $1 \times 10^{16} / \mathrm{cm}^{3}$. The theoretical prediction fits the experiment well at drift fields less than $-0.74 \mathrm{kV} / \mathrm{cm}$, which confirms the individually measured $\mathrm{LiNbO}_{3}$ and $\mathrm{In}_{0.53} \mathrm{Ga}_{0.47} \mathrm{As}$ material parameters. However, the model fails at larger drift fields, indicating that the experimental gain rollover cannot be captured with a model, such as that from Eq. (1), that is valid only for small signal, steady- 
a

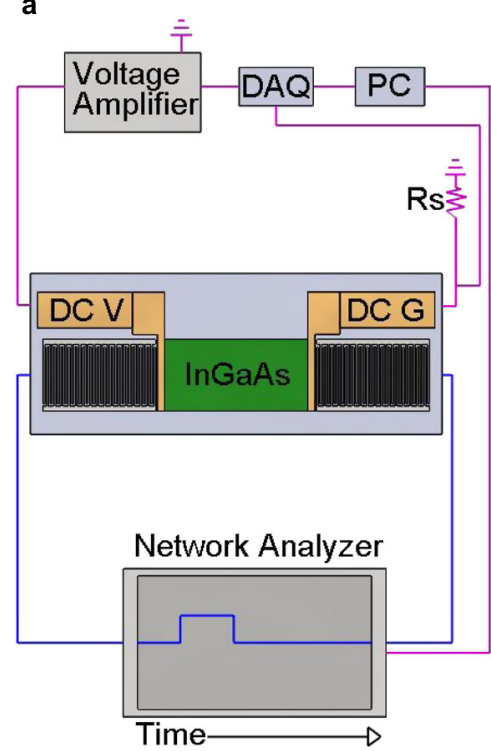

b
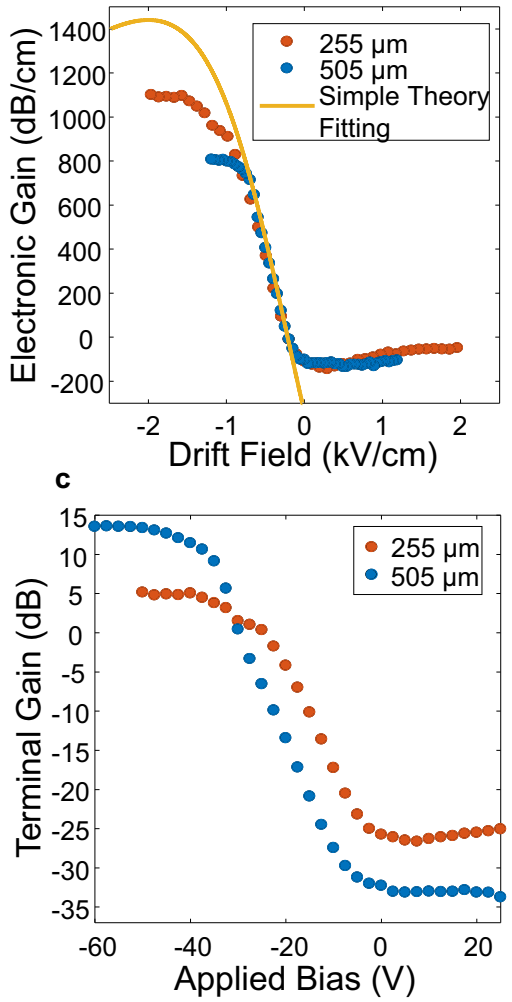
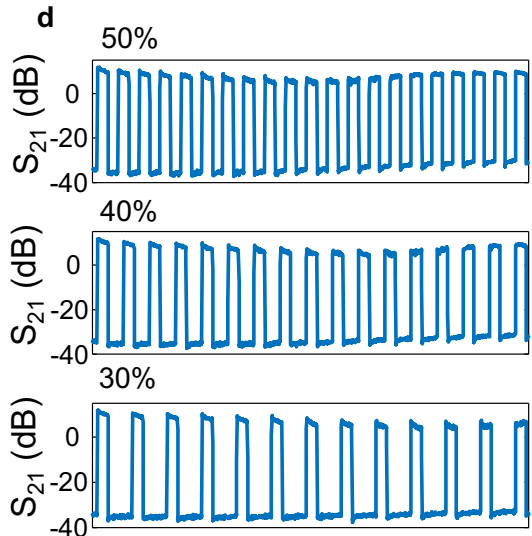

$20 \%$

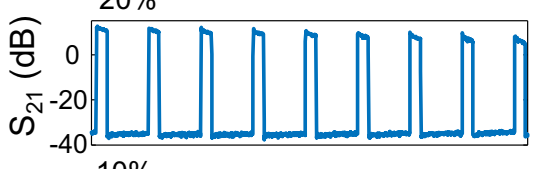

$10 \%$

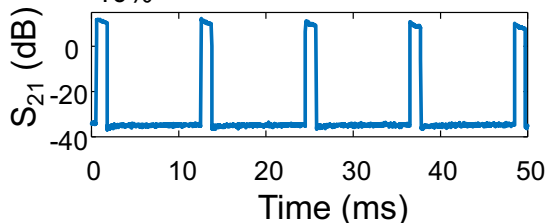

Fig. 4 Acoustoelectric amplifier. a Schematic of the experimental setup for gain measurements consisting of a computer (PC), network analyzer, voltage amplifier, data acquisition system (DAQ), and shunt resistor (Rs). DC V and DC G refer to the applied DC bias and ground, respectively. $\mathbf{b}$ Measured experimental gain as a function of drift field with a comparison to the expected electronic gain from Eq. (1). c Terminal gain as a function of applied bias. A terminal gain of $13.5 \mathrm{~dB}$ is achieved at $-60 \mathrm{~V}$ applied bias for a $505 \mu \mathrm{m}$ long amplifier device. d Experimental gain pulses with increasing applied bias duty cycles from $10 \%$ to $50 \%$.

state conditions where the material parameters are spatially uniform and do not change with voltage or temperature. The $255 \mu \mathrm{m}$ long device has a maximum electronic gain of $1101 \mathrm{~dB} / \mathrm{cm}$ at a drift field of $-2 \mathrm{kV} / \mathrm{cm}$ and the $505 \mu \mathrm{m}$ long device has a maximum electronic gain of $806 \mathrm{~dB} / \mathrm{cm}$ at a drift field of $-1.2 \mathrm{kV} / \mathrm{cm}$. Although we demonstrate the largest electronic gains per unit length for any known acoustoelectric material platform, the performance of these devices is limited by thermal effects and can be further improved to operate continuously and reach the true peak acoustoelectric gain by engineering the system to dissipate heat more effectively.

The terminal gain of the acoustoelectric amplifier with increasing duty cycles was examined in order to further assess the acoustoelectric device performance. A plot of terminal gain in $\mathrm{dB}$ as a function of applied bias for a $1 \mathrm{~ms}$ voltage pulse is shown in Fig. 4c, for the $505 \mu \mathrm{m}$ long acoustoelectric amplifier. A terminal gain of $13.5 \mathrm{~dB}$ is obtained at an applied bias of $-60 \mathrm{~V}$, corresponding to a dissipated DC power of only $15 \mathrm{~mW}$. The thermal stability of the acoustoelectric amplifier was investigated by increasing the duty cycle of the $1 \mathrm{~ms}$ voltage pulse from $10 \%$ to $50 \%$ during a $50 \mathrm{~ms}$ measurement cycle. Gain measurements with respect to time, taken during the application of the voltage pulses, are shown in Fig. $4 \mathrm{~d}$ for the increasing duty cycles. The gain remains stable at a $50 \%$ duty cycle, with over $10 \mathrm{~dB}$ of terminal gain demonstrated.

We also measured the acoustic output power and DC to RF acoustic power efficiency as a function of the DC power dissipation and acoustic input power (see Supplementary Note 4 and Supplementary Fig. 4). The power efficiency $(\eta)$ is defined as $\eta=\frac{P_{\mathrm{OUT}}^{A}}{P_{\mathrm{DISS}}^{\mathrm{DC}}}$ where $P_{\mathrm{OUT}}^{A}$ is the acoustic output power and $P_{\mathrm{DISS}}^{\mathrm{DC}}$ is the dissipated DC power. We achieve an $\eta$ of $4.4 \%$ at an acoustic input power of $-34 \mathrm{dBm}$ and a DC power dissipation of $12 \mathrm{~mW}$. Ultimately $\eta$ is limited by the fact that signal saturation of an acoustic amplifier fundamentally occurs when all the electrons available for the acoustoelectric interaction are incorporated into the acoustoelectrically induced RF current, saturating the gain ${ }^{39}$. The saturation output power increases with $N$, but this will also increase $P_{\mathrm{DISS}}^{\mathrm{DC}}$. Depending on device requirements, $\eta$ can potentially be optimized by exploring the parameter space of $N$, $\sigma t$, and RF saturation power with device designs that prioritize efficient thermal dissipation. The noise figure of acoustoelectric amplifiers has been discussed in previous works and it was found that trapping effects in the semiconductor are the dominant source of noise ${ }^{40}$. Devices using evaporated semiconductor materials had a defectivity-limited noise figure of $9 \mathrm{~dB}^{41}$. In the limit of direct contact between the semiconductor and piezoelectric and very small trap density, as expected for an MOCVD film, the theoretical noise figure for the experimental values in this work is $1 \mathrm{~dB}$, under the assumption that trapping effects are the dominant noise source (see Supplementary Note 5).

The critical obstacles to integrate acoustoelectric devices into future RFSPs are stable operations with a continuously applied DC bias, in addition to increasing the RF input power dynamic range and overall $\mathrm{RF}$ power handling. Recent experiments show that, by using a $\mathrm{LiNbO}_{3}$ thin film on a Si substrate (as opposed to a bulk $\mathrm{LiNbO}_{3}$ substrate) that still supports guided acoustic waves with and without the amplifier material, the heat dissipation efficiency drastically improves via the $30 \mathrm{X}$ improvement in the thermal conductivity of $\mathrm{Si}$ compared to $\mathrm{LiNbO}_{3}{ }^{42,43}$. The device 
fabrication remains identical except for replacing the $\mathrm{LiNbO}_{3}$ substrate with a $\mathrm{LiNbO}_{3}$ on Si substrate. In a $250 \mu \mathrm{m}$ long device, we achieve an electronic gain of $15.4 \mathrm{~dB}$, while operating continuously and output power of $8.7 \mathrm{dBm}$ (see Supplementary Note 6 and Supplementary Fig. 5). These initial results suggest that the $\mathrm{LiNbO}_{3}$ film on bulk Si substrate is a promising path forward to overcome the challenges of thermal management and power handling for active acoustic wave devices, and the material and device parameters will be further optimized in our future work.

Acoustoelectric circulator. As discussed above, the acoustoelectric effect is inherently nonreciprocal. Thus, a circulator can in principle be formed by incorporating the effect into a threeport device with proper management of the direction of gain between each port. A schematic of the developed three-port acoustoelectric circulator is shown in Fig. 5a. Reversing multistrip couplers (RMSCs) ${ }^{44,45}$ serve to transmit surface acoustic waves in a designed frequency band from an upper track to a lower track, while simultaneously reversing the propagation direction. These RMSCs consist of periodic metal electrodes that are interlaced between two spatially distinct but parallel channels. Due to the connections between electrodes in the upper and lower channels, the complex electric potentials that are generated in the electrodes of one channel of the RMSC by the SAW generate potentials in the other channel that excite a SAW propagating in the opposite direction. RMSCs have been previously demonstrated for acoustic ring filters, as shown in Fig. 5b, where they function to transmit the SAW energy generated at an IDT in an upper track to be detected at an IDT in a lower track with minimal acoustic losses ${ }^{46}$.

For the acoustoelectric circulator demonstrated here, at each of the three IDTs, which form the three ports of the circulator, an electrical signal can be converted to an acoustic wave by the inverse piezoelectric effect. This acoustic wave is then amplified only for one helicity depending on the sign and magnitude of the applied drift field. Simultaneously, the acoustic wave for the opposite helicity is attenuated. Here helicity defines the two ways that the SAW can travel around the loop of the three ports connected by the RMSCs. One helicity then defines counterclockwise propagation of the SAW while the other helicity defines clockwise SAW propagation. Once the acoustic wave reaches the sequential port's IDT, it is converted back into an electrical signal by the piezoelectric effect. The theoretical nonreciprocity as a function of applied bias for each acoustoelectric section of the device is shown in Fig. 5c. As can be seen, the contrast ratio between forward and backward propagating waves increases with applied bias. We then seek to achieve an acoustoelectric circulator with low insertion loss and large isolation while maintaining low dissipated DC power.
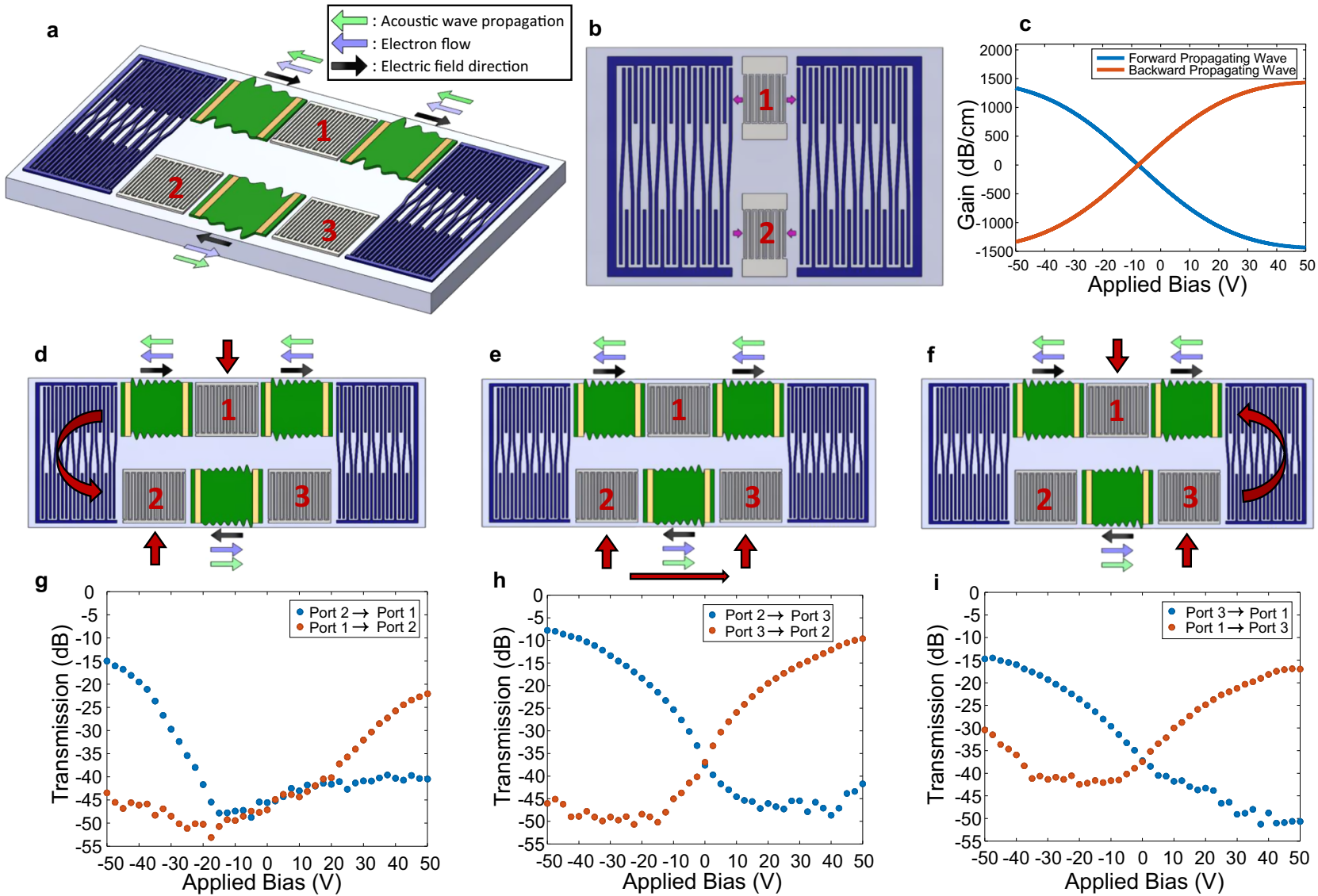

Fig. 5 Acoustoelectric circulator. Schematics of the (a) three-port acoustoelectric circulator and (b) two-port ring filter that is the baseline for the device design. The $\mathrm{In}_{0.53} \mathrm{Ga}_{0.47} \mathrm{As}$ is shown in green, the $\mathrm{LiNbO}_{3}$ substrate in light gray, the RF IDTs in dark gray, the DC contacts in gold, and the couplers in purple. The light green, light purple, and black arrows denote the directions for the acoustic wave propagation, electron flow, and electric field direction, respectively, for the counterclockwise helicity. c Theoretical nonreciprocal gain as a function of applied bias for each acoustoelectric section. Schematics of the measurements done from (d) port 1 to port 2, (e) port 2 to port 3, and (f) port 3 to port 1 with the corresponding experimental data of acoustic transmission as a function of an applied pulsed DC bias shown in ( $(\mathbf{g}),(\mathbf{h})$, and (i), respectively. The acoustic transmission is measured by detecting a change in the S-parameters with respect to time during a continuous RF input at $276 \mathrm{MHz}$ and the application of a 1 ms voltage pulse. A measurement is made every 50 ms giving a duty cycle of $2 \%$. 


\begin{tabular}{|c|c|c|c|}
\hline Ports & Insertion loss (dB) & Isolation (dB) & Helicity \\
\hline $1 \rightarrow 2$ & 22 & 41 & Counter-clockwise \\
\hline $2 \rightarrow 3$ & 8 & 46 & Counter-clockwise \\
\hline $3 \rightarrow 1$ & 15 & 30 & Counter-clockwise \\
\hline $1 \rightarrow 3$ & 17 & 51 & Clockwise \\
\hline $3 \rightarrow 2$ & 10 & 42 & Clockwise \\
\hline $2 \rightarrow 1$ & 15 & 44 & Clockwise \\
\hline
\end{tabular}

Figure $5 \mathrm{~d}-\mathrm{f}$ shows each pair of ports characterized sequentially beginning with ports 1 and 2 (Fig. 5d), then ports 2 and 3 (Fig. 5e), followed by ports 3 and 1 (Fig. 5f). The measured Sparameters for ports 1 and 2 , ports 2 and 3 , and ports 3 and 1 are shown in Fig. 5g-i, respectively, as a function of the applied bias. The S-parameters are measured with respect to time during the application of a continuous $\mathrm{RF}$ signal at $276 \mathrm{MHz}$ and a voltage pulse of $1 \mathrm{~ms}$. The transmission plotted in Fig. $5 \mathrm{~g}-\mathrm{i}$, is then the Sparameter values measured when the voltage pulse is on. As expected, the experimental contrast ratio increases with increasing applied bias, leading to smaller insertion loss and larger isolation. A summary of the acoustoelectric circulator performance for counter-clockwise and clockwise helicities is given in Table 1. The insertion loss and isolation are the S-parameter values taken from Fig. $5(\mathrm{~g}-\mathrm{i})$ at an applied bias of $\pm 50 \mathrm{~V}$. From port 2 to port 3 , an insertion loss of $8 \mathrm{~dB}$ is obtained with an isolation of $46 \mathrm{~dB}$. While this is the highest performing pair of ports, it is expected that the other pairs of ports will show improved performance with optimization of the RMSCs.

We have demonstrated a proof-of-concept acoustoelectric circulator where the functionality of the device can be modified, depending on the application, by changes to the ports and routing, enabling a novel class of nonreciprocal acoustic wave devices. While our acoustoelectric circulator provides a significantly improved device footprint and a better path forward for on-chip integration compared to commercial circulators that require ferrite-based magnetic materials, further improvements are required to reduce insertion losses, reduce DC power dissipation, enable continuous operation, and improve RF power handling and device linearity. The data shown in Fig. 5 is taken with a continuous RF power input, but a pulsed DC bias. As discussed above in the context of the amplifier, recently we have found that our acoustoelectric devices can operate with a continuously applied DC bias through improved thermal management with a $\mathrm{LiNbO}_{3}$ on $\mathrm{Si}$ substrate due to the $30 \mathrm{X}$ larger thermal conductivity of $\mathrm{Si}$ compared to $\mathrm{LiNbO}_{8}$ (see Supplementary Note 6 and Supplementary Fig. 5). While the linearity of modulated circulator schemes depends primarily on the RF switch size ${ }^{47}$, for our acoustoelectric circulator the linearity depends on the RF power handling of the device and the onset of signal compression, which is fundamentally limited by the semiconductor carrier concentration. Therefore, the improved heat dissipation with a $\mathrm{LiNbO}_{3}$ on $\mathrm{Si}$ substrate also increases the $\mathrm{RF}$ power handling, and therefore the device linearity and dynamic range (see Supplementary Note 6 and Supplementary Fig. 5). Insertion loss can be improved from ports 1 to 2 and ports 3 to 1 by optimization of the RMSC design (see Supplementary Note 7 and Supplementary Fig. 6 for the experimental Sparameters for a metal-only three-port ring filter). Insertion losses can also be significantly improved by exploring different geometries and material stacks of the DC contacts to reduce acoustic reflections. In addition, improvements to the acoustoelectric performance through thermal management and further reducing $\sigma t$ will also further optimize the circulator.

\section{Discussion}

Here we have demonstrated the fabrication of passive acoustic delay lines on the same chip as the highest-performing acoustic wave amplifiers ever demonstrated and the first-ever demonstration of an acoustoelectric circulator. This was enabled through our heterogeneously integrated material platform of a $50 \mathrm{~nm}$ epitaxial $\mathrm{In}_{0.53} \mathrm{Ga}_{0.47} \mathrm{As}$ semiconductor with low carrier concentration and large mobility on a $41^{\circ} \mathrm{YX} \mathrm{LiNbO}_{3}$ piezoelectric substrate, supporting an SH-SAW mode with a $K^{2}$ of $17 \%$. We have demonstrated SH-SAW acoustic delay line filters with an insertion loss of $8.4 \mathrm{~dB}$ and fractional bandwidth of $4.1 \%$, acoustic amplification with $13.5 \mathrm{~dB}$ of terminal gain with $15 \mathrm{~mW}$ of dissipated DC power, and an acoustoelectric circulator with an isolation of $46 \mathrm{~dB}$.

These experimental demonstrations suggest a promising path forward to develop an all-acoustic and therefore ultra-compact RFSP utilizing the acoustoelectric effect. The fundamental components of a conventional simultaneous transmit and receive (STAR) RFSP for a single frequency band are shown in Fig. 6a. The circulator is implemented in a ferrite material; passive filtering is done with piezoelectric acoustic wave technology; and the power amplifier (PA), low noise amplifier (LNA), local oscillator (LO), and mixer are implemented with CMOS and compound semiconductor electronics. Many modern RF devices, such as Long Term Evolution (LTE) smartphones, require separate RFSPs for more than 30 different frequency bands. As each band needs at least two filters, two mixers, one LO, one PA, and one LNA, this means that more than 60 filters and mixers are required in addition to more than $30 \mathrm{LOs}$, PAs, and LNAs ${ }^{16}$

Figure $6 \mathrm{~b}$ shows a concept for the RFSP of Fig. 6a, entirely in the acoustic wave domain. Passive delay line and filtering components are achieved through interactions with periodic electrodes and RMSCs that serve as track changers ${ }^{44}$. All active, nonlinear, and nonreciprocal functions are provided by the acoustoelectric interaction wherever semiconductor material remains after processing. In particular, the LNA and PA are achieved through the addition of acoustic amplification, while the mixer is achieved by the enhanced nonlinearity from electronphonon coupling, which has previously been applied for convolution $^{48-50}$ and correlation ${ }^{51-53}$. The combination of RMSC track changers for routing with the acoustoelectric effect enables an acoustic circulator and LO, as an LO can be viewed as a circulator in which the gain exceeds the round-trip acoustic losses, inducing self-oscillation at the frequency of highest gain. At $276 \mathrm{MHz}$, the approximate size of the RF acoustics chip in Fig. 6b would be $4.5 \mathrm{~mm} \times 1 \mathrm{~mm}$. For the common LTE band occurring at approximately $1.9 \mathrm{GHz}$, the smaller acoustic wavelength reduces this size to approximately $0.65 \mathrm{~mm} \times 0.15 \mathrm{~mm}$. In addition to a drastically reduced footprint and improved on-chip device integration, with an all-acoustic platform, there is an increased opportunity to implement signal processing functions passively through the design of the IDT transfer function, such as dispersion control and correlation. This could reduce the required functionality and power requirements of analog-to-digital converters and digital signal processors for applications that are constrained by cost, power, and size requirements. Therefore, this approach could also lead to better device performance and reduced power consumption in addition to the significantly reduced footprint and improved on-chip integration.

Regarding the goal of achieving an all-acoustic, STAR RF front-end, we have experimentally demonstrated three critical components: passive filtering, a high gain amplifier that could be implemented as an LNA, and an acoustic wave circulator. The remaining necessary components are a $\mathrm{PA}, \mathrm{LO}$, and frequency mixer. While not achieved in this work, their development should be extensible functionalities of the devices demonstrated here. 
a

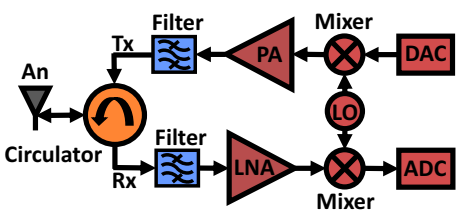

$\square$ Ferrite $\square$ Piezoelectric Acoustic Wave

$\square$ CMOS or Compound Semiconductor

b
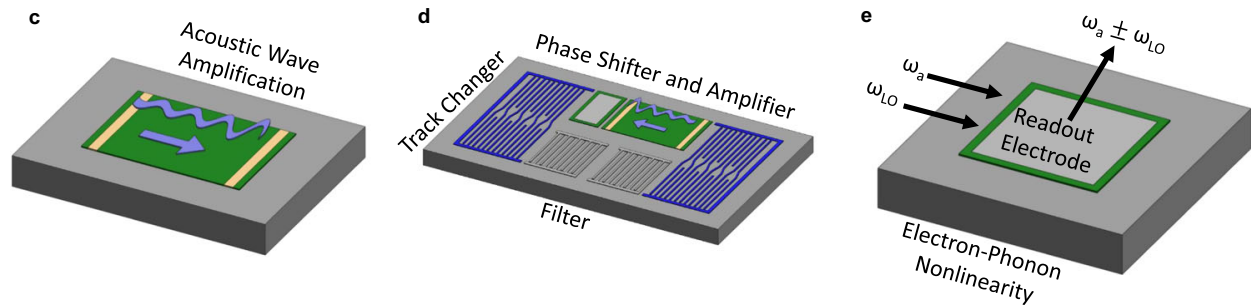

Fig. 6 RFSP overview and components of an all-acoustic RFSP. a Fundamental components of a multi-chip approach to an RF front end capable of STAR. The $T x$ and Rx paths are connected to the antenna (An) through a circulator. The Tx path requires a digital-to-analog converter (DAC) while the Rx path requires an analog-to-digital converter (ADC). b Schematic of an all-acoustic RF front end. The $\ln _{0.53} \mathrm{Ga}_{0.47} \mathrm{As}$ is shown in green, the LiNbO ${ }_{3}$ substrate in light gray, DC contacts in gold, aluminum filters in dark gray, and track changers in dark purple. Arrows in light purple indicate the direction of acoustic wave propagation. c A PA utilizes the acoustoelectric effect for acoustic wave amplification in a device design optimized for large RF power handling. $\mathbf{d}$ The LO is constructed from RMSCs, an acoustic filter, an acoustic amplifier, and an acoustic wave phase shifter. e An acoustic wave mixer could operate by the nonlinearity from the electron-phonon coupling.

A schematic of a PA is shown in Fig. 6c. While the structure is essentially the same as the acoustic amplifier demonstrated in this work, a PA will require modifications for increased RF power handling. This will likely require both improved thermal dissipation through device design and the addition of an epitaxial layer with increased carrier concentration, $N$, to increase the RF saturation power ${ }^{39}$. In addition, carrier concentration could be varied for different acoustoelectric devices on the same wafer by the inclusion of gate electrodes that control the local carrier concentration with a bias field perpendicular to the acoustic wave propagation.

A notional LO, as shown in Fig. 6d, could be implemented through the inclusion of an acoustic amplifier in a two-port ring filter optimized for low insertion loss, such that the round-trip gain exceeds the round-trip loss. A LO is then similar in function to the circulator demonstrated here and simply requires modification of the track changer path. As described above, gate electrodes could be included to form a phase shifter as a part of the LO. Due to the acoustoelectric interaction, tuning the carrier concentration, $N$, via application of a gate voltage leads to a change in the SAW velocity, which results in a phase shift.

Finally, a mixer, as shown in Fig. 6e, could be developed by extending previous acoustoelectric correlator demonstrations that utilize nonlinear frequency mixing ${ }^{51-53}$. Two SAWs, one at the $\mathrm{RF}$ acoustic frequency $\omega_{a}$ and the other at the LO frequency $\omega_{\mathrm{LO}}$, co-propagate along the surface of the acoustoelectric heterostructure. Frequency mixing between the two SAWs occurs through the nonlinear electron-phonon interaction ${ }^{48,54,55}$. A readout electrode could then be designed and implemented to pull out the desired signal at the upconverted or downconverted frequency.

Potential additional applications of this material platform include acoustic switch networks and the amplification of phonons in combination with $\mathrm{LiNbO}_{3}$ piezoelectric optomechanical devices ${ }^{56,57}$. Devices of this kind could also be utilized in future chip-scale systems for radiofrequency signal processing that use Brillouin interactions in integrated photonics. Given that the gain in Brillouin amplifiers and lasers is inversely proportional to phonon loss rates, the ability to actively control these (and reduce them to zero) could lead to unprecedented performance and novel functionality in these systems ${ }^{58}$. Finally, because the performance of these compound semiconductor devices is expected to improve at cryogenic temperatures ${ }^{59}$, these acoustoelectric devices may be able to play the role of circulators and low-noise, high-gain amplifiers for quantum phononic devices ${ }^{60-62}$, perhaps playing a role analogous to the Josephson parametric amplifier ${ }^{63}$, which has been used for the readout of single microwave photons in superconducting circuit-based quantum computing.

\section{Data availability}

The datasets generated during and analyzed during this study are available from the corresponding author on reasonable request.

Received: 11 September 2020; Accepted: 7 April 2021;

Published online: 13 May 2021 


\section{References}

1. Morita, T., Watanabe, Y., Tanaka, M. \& Nakazawa, Y. Wide-band low-loss double-mode SAW filters. Ultrason.1, 95-104 (1992).

2. Yamanouchi, K. \& Furuyashiki, H. New Low-loss SAW filter using internal floating electrode reflection types of single-phase unidirectional transducer. Electron Lett. 20, 989-990 (1984).

3. Yang, Y. S., Lu, R. C., Gao, L. Q. \& Gong, S. B. 4.5 GHz lithium niobate MEMS filters With $10 \%$ fractional bandwidth for $5 \mathrm{G}$ front-ends. J. Microelectromech. Syst. 28, 575-577 (2019).

4. Lobl, H. P. et al. Materials for bulk acoustic wave (BAW) resonators and filters. J. Eur. Ceram. Soc. 21, 2633-2640 (2001).

5. Loebl, H. P. et al. Piezoelectric thin AlN films for bulk acoustic wave (BAW) resonators. Mater. Chem. Phys. 79, 143-146 (2003).

6. Linten, D. et al. A 5-GHz fully integrated ESD-protected low-noise amplifier in 90-nm RF CMOS. IEEE J. Solid-State Circ. 40, 1434-1442 (2005).

7. Reynaert, P. \& Steyaert, M. S. J. A $1.75-\mathrm{GHz}$ polar modulated CMOS RF power amplifier for GSM-EDGE. IEEE J. Solid-State Circ. 40, 2598-2608 (2005).

8. Hassan, M., Larson, L. E., Leung, V. W., Kimball, D. F. \& Asbeck, P. M. A wideband CMOS/GaAs HBT envelope tracking power amplifier for 4G LTE mobile terminal applications. IEEE Trans. Micro. Theory Tech. 60, 1321-1330 (2012).

9. Maas, S. A. A GaAs-MESFET mixer with very low intermodulation. IEEE T Micro. Theory Tech. 35, 425-429 (1987).

10. Fay, C. E. \& Comstock, R. L. Operation of ferrite junction circulator. IEEE T Micro. Theory Tech. 13, 15-1 (1965).

11. Pardavi-Horvath, M. Microwave applications of soft ferrites. J. Magn. Magn. Mater. 215, 171-183 (2000).

12. Estep, N. A., Sounas, D. L., Soric, J. \& Alu, A. Magnetic-free non-reciprocity and isolation based on parametrically modulated coupled-resonator loops. Nat. Phys. 10, 923-927 (2014).

13. Fleury, R., Sounas, D. L. \& Alu, A. Subwavelength ultrasonic circulator based on spatiotemporal modulation. Phys. Rev. B 91, 174306 (2015).

14. Torunbalci, M. M., Odelberg, T. J., Sridaran, S., Ruby, R. C. \& Bhave, S. A. An FBAR circulator. IEEE Micro. Wirel. Co. 28, 395-397 (2018).

15. Yu, Y. et al. Magnetic-free radio frequency circulator based on spatiotemporal commutation of MEMS Resonators. In 2018 IEEE Micro Electro Mechanical Systems (MEMS) Proc. IEEE Micr. Elect. 154-157 (2018).

16. Mahon, S. The $5 \mathrm{G}$ effect on RF filter technologies. IEEE Trans. Semicond. Manufactur. 30, 494-499 (2017).

17. Bhushan, N. et al. Network densification: the dominant theme for wireless evolution into 5G. IEEE Commun. Mag. 52, 82-89 (2014).

18. Bogucka, H., Kryszkiewicz, P. \& Kliks, A. Dynamic spectrum aggregation for future 5G communications. IEEE Commun. Mag. 53, 35-43 (2015).

19. Shinjo, S., Nakatani, K., Tsutsumi, K. \& Nakamizo, H. Integrating the front end: a highly integrated RF front end for high-SHF wide-band massive MIMO in 5G. IEEE Micro. Mag. 18, 31-40 (2017).

20. Pehlke, D. R. \& Walsh, K. LTE-advanced pro RF front-end implementations to meet emerging carrier aggregation and DL MIMO requirements. IEEE Commun. Mag. 55, 134-141 (2017).

21. Chen, $X$. et al. High performance lithium niobate surface acoustic wave transducers in the 4-12 GHz super high frequency range. J. Vac. Sci. Technol. $B$ 33, 06F401 (2015).

22. Lu, R. C., Manzaneque, O. M., Yang, Y. S., Li, M. H. \& Gong, S. B. Gigahertz low-loss and wideband S0 mode lithium niobate acoustic delay lines. IEEE Trans. Ultrason. Ferr. 66, 1373-1386 (2019).

23. Lu, R. C., Yang, Y. S., Li, M. H., Breen, M. \& Gong, S. B. 5-GHz antisymmetric mode acoustic delay lines in lithium niobate thin film. IEEE Trans. Micro. Theory 68, 573-589 (2020).

24. Coldren, L. A. \& Kino, G. S. Monolithic acoustic surface-wave amplifier. Appl. Phys. Lett. 18, 317-31 (1971).

25. Collins, J. H., Lakin, K. M., Quate, C. F. \& Shaw, H. J. Amplification of acoustic surface waves with adjacent semiconductor and piezoelectric crystals. Appl. Phys. Lett. 13, 314-31 (1968).

26. Kino, G. S. Acoustoelectric interactions in acoustic-surface-wave devices. Proc. IEEE 64, 724-748 (1976).

27. White, R. M. Surface elastic-wave propagation and amplification. IEEE Trans. Electron Dev. Ed14, 181-18 (1967).

28. Bhaskar, U. K., Bhave, S. A. \& Weinstein, D. Silicon acoustoelectronics with thin film lithium niobate. J. Phys. D 52, 05LT01 (2018).

29. Carmichael, C. P., Smith, M. S., Weeks, A. R. \& Malocha, D. C. Experimental investigation of surface acoustic wave acoustoelectric effect using a graphene film on lithium niobate. IEEE Trans. Ultrason Ferr. 65, 2205-2207 (2018).

30. Yamanouchi, K. \& Shibayama, K. Propagation and amplification of rayleigh waves and piezoelectric leaky surface-waves in $\mathrm{LiNbO}_{3}$. J. Appl Phys. 43, 856-85 (1972)

31. Kino, G. S. \& Reeder, T. M. Normal mode theory for rayleigh wave amplifier. IEEE Trans. Electron Dev. Ed18, 909-90 (1971).
32. Kompfner, R. The traveling-wave tube as amplifier at microwaves. Proc. IRE 35, 124-127 (1947).

33. Kuzmany, H. Acoustoelectric interaction in semiconductors. Phys. Status Solidi A 25, 9-67 (1974).

34. Spector, H. N. Amplification of acoustic waves through interaction with conduction electrons. Phys. Rev. 127, 1084-108 (1962).

35. Adler, R. Simple theory of acoustic amplification. IEEE T Son. Ultrason Su18, 115-11 (1971).

36. Hackett, L. et al. High-gain leaky surface acoustic wave amplifier in epitaxial InGaAs on lithium niobate heterostructure. Appl. Phys. Lett. 114, 253503 (2019).

37. Smith, W. R., Gerard, H. M., Collins, J. H., Reeder, T. M. \& Shaw, H. J. Analysis of interdigital surface wave transducer by use of an equivalent circuit model. IEEE Trans. Micro. Theory Mt17, 856-85 (1969).

38. Koskela, J., Knuuttila, J. V., Makkonen, T., Plessky, V. P. \& Salomaa, M. M. Acoustic loss mechanisms in leaky SAW resonators on lithium tantalate. IEEE T Ultrason Ferr. 48, 1517-1526 (2001).

39. Tien, P. K. Nonlinear theory of ultrasonic wave amplification and current saturation in piezoelectric semiconductors. Phys. Rev. 171, 970-97 (1968)

40. Kino, G. S. \& Coldren, L. A. Noise figure calculation for Rayleigh-Wave amplifier. Appl. Phys. Lett. 22, 50-52 (1973).

41. Coldren, L. A. \& Kino, G. S. InSb on a piezoelectric Rayleigh-wave amplifier. IEEE Trans. Electron Dev. Ed21, 421-427 (1974).

42. Glassbrenner, C. J. \& Slack, G. A. Thermal conductivity of silicon + germanium from 3 degrees K to melting point. Phys. Rev. 134, 1058-105 (1964).

43. Wong, K.-K. Properties of Lithium Niobate. No. 28. (IET, 2002).

44. Danicki, E. Reversing multistrip coupler. Ultrasonics 31, 421-424 (1993).

45. Pollock, W. et al. Filter using single-phase IDT and no external tuning. IEEE Trans. Son. Ultrason 32, 113-113 (1985).

46. Dobershtein, S. A. \& Malyukhov, V. A. SAW ring filters with insertion loss of 1 dB. IEEE Trans. Ultrason Ferr. 44, 590-596 (1997).

47. Yu, Y. \& Rinaldi, M. Micro-acoustic quad circulator with self-interference cancellation. 2020 IEEE 33rd International Conference on Micro Electro Mechanical Systems (MEMS). 1270-1273 (2020).

48. Lee, C. W. \& Gunshor, R. L. Nonlinear-interaction of acoustic surface-waves from coupling to charge-carriers. J. Appl. Phys. 44, 4807-4812 (1973).

49. Solie, L. P. Acoustic surface-wave convolver with bidirectional amplification. Appl. Phys. Lett. 25, 7-10 (1974).

50. Wang, W. C. \& Das, P. Surface-wave convolution in piezoelectric semiconductors. Pr. Inst. Electr. Elect. 60, 1109-110 (1972).

51. Bers, A. \& Cafarell, J. H. Surface state memory in surface acoustoelectric correlator. IEEE Trans. Electron Dev. Ed21, 744-744 (1974).

52. Ingebrigtsen, K. A. Schottky diode acoustoelectric memory and correlatornovel programmable signal processor. Proc. IEEE 64, 764-769 (1976).

53. Ralston, R. W., Hurlburt, D. H., Leonberger, F. J., Cafarellia, J. H. \& Stern, E. New signal-processing device, integrating correlator. IEEE Trans. Son. Ultrason 25, 244-244 (1978).

54. Conwell, E. M. \& Ganguly, A. K. Mixing of acoustic waves in piezoelectric semiconductors. Phys. Rev. B 4, 2535-253 (1971).

55. Lee, C. W. \& Gunshor, R. L. Enhancement of nonlinearity in surface-acousticwave propagation from coupling to charge-carriers. Appl. Phys. Lett. 20, 288-28 (1972).

56. Jiang, W. T. et al. Efficient bidirectional piezo-optomechanical transduction between microwave and optical frequency. Nat. Commun. 11, 1-7 (2020).

57. Shao, L. B. et al. Microwave-to-optical conversion using lithium niobate thinfilm acoustic resonators. Optica 6, 1498-1505 (2019).

58. Eggleton, B. J., Poulton, C. G., Rakich, P. T., Steel, M. J. \& Bahl, G. Brillouin integrated photonics. Nat. Photon. 13, 664-677 (2019).

59. Hornibrook, J. M. et al. Cryogenic control architecture for large-scale quantum computing. Phys. Rev. Appl. 3, 4010-4010 (2015).

60. Forsch, M. et al. Microwave-to-optics conversion using a mechanical oscillator in its quantum ground state. Nat. Phys. 16, 69-74 (2020).

61. Mirhosseini, M., Sipahigil, A., Kalaee, M. \& Painter, O. J. Superconducting qubit to optical photon transduction. Nature 588, 559-603 (2020).

62. Arrangoiz-Arriola, P. et al. Coupling a superconducting quantum circuit to a phononic crystal defect cavity. Phys. Rev. X 8, 031007 (2018).

63. Yamamoto, T. et al. Flux-driven Josephson parametric amplifier. Appl. Phys. Lett. 93, 042510 (2008)

\section{Acknowledgements}

This work was supported by the Laboratory Directed Research and Development program at Sandia National Laboratories, a multimission laboratory managed and operated by National Technology and Engineering Solutions of Sandia LLC, a wholly-owned subsidiary of Honeywell International Inc. for the U.S. Department of Energy's National Nuclear Security Administration under contract DE-NA0003525. M.E. performed this work, in part, at the Center for Integrated Nanotechnologies, an Office of Science User Facility operated for the U.S. Department of Energy Office of Science. This paper 
describes objective technical results and analysis. Any subjective views or opinions that might be expressed in the paper do not necessarily represent the views of the U.S. Department of Energy or the United States Government.

\section{Author contributions}

L.H. and M.E. came up with the device concepts and experimental implementations. L.H., M.M., D.D., A.T., T.F., and M.E. designed the devices and fabrication process flow. M.M., G.P., and S.A. fabricated the devices. L.H. and F.B. performed the measurements. L.H. and M.E. analyzed all data. All authors have given approval to the final version of the manuscript.

\section{Competing interests}

The authors declare no competing interests.

\section{Additional information}

Supplementary information The online version contains supplementary material available at https://doi.org/10.1038/s41467-021-22935-1.

Correspondence and requests for materials should be addressed to M.E.

Peer review information Nature Communications thanks the anonymous reviewer(s) for their contribution to the peer review of this work. Peer reviewer reports are available.
Reprints and permission information is available at http://www.nature.com/reprints

Publisher's note Springer Nature remains neutral with regard to jurisdictional claims in published maps and institutional affiliations.

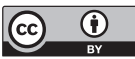

Open Access This article is licensed under a Creative Commons Attribution 4.0 International License, which permits use, sharing, adaptation, distribution and reproduction in any medium or format, as long as you give appropriate credit to the original author(s) and the source, provide a link to the Creative Commons license, and indicate if changes were made. The images or other third party material in this article are included in the article's Creative Commons license, unless indicated otherwise in a credit line to the material. If material is not included in the article's Creative Commons license and your intended use is not permitted by statutory regulation or exceeds the permitted use, you will need to obtain permission directly from the copyright holder. To view a copy of this license, visit http://creativecommons.org/ licenses/by/4.0/.

This is a U.S. Government work and not under copyright protection in the US; foreign copyright protection may apply 2021 